SHORT REPORT

\title{
Cervical smears and human papillomavirus typing in sex workers
}

\section{R Mak, L Van Renterghem, C Cuvelier}

Sex Transm Infect 2004;80:118-120. doi: 10.1136/sti.2002.003749

\begin{abstract}
Objectives: Sex workers are at increased risk for sexually transmitted infections (STI), human papillomavirus (HPV) and hence cervical cancer. In Belgium screening for cervical cancer starts at the age of 25 , and is at 3 yearly intervals. The aim of this study is to assess risks for abnormal cervical smears and HPV in sex workers and decide whether the current screening policy is sufficient for them.

Methods: In an outreach programme for sex workers results of 653 smears sampled between 1992 and 2001 were analysed, and compared to a control group matched for age from the general population in 2001. Separately, 99 consecutive samples were typed for HPV and compared to an equal control group, matched for age. Smears and typing were performed according to current techniques.

Results: In the sex worker group $2.6 \%$ were diagnosed with atypical glandular cells of undetermined significance (AGUS)/atypical squamous cells of undetermined significance (ASCUS), $15.6 \%$ with low grade squamous intraepithelial lesion (LSIL), and $2.9 \%$ with high grade squamous intraepithelial lesion (HSIL), and in the control group results were $1.4 \%, 2.9 \%(p<0.001)$ and $0.6 \%(p<0.001)$ respectively. When considering only those under 25 years, $24.4 \%$ should have further follow up. Of the sex workers, $77.4 \%$ were positive for one or more types of HPV $155.9 \%$ for high risk HPV), in comparison with $27.6 \%$ of the control group (14.3\% for high risk HPV) $(p<0.001)$. In high risk HPV samples more LSIL and HSIL were found.

Conclusion: Abnormal smears and high risk HPV were significantly more prevalent in sex workers than in controls. Current screening policy would miss many sex workers with an abnormal smear who should be referred for further follow up. It is proposed to screen sex workers when they enter prostitution regardless of their age.
\end{abstract}

$\mathrm{H}$ uman papillomavirus (HPV) is sexually transmitted and causally related to cervical cancer, ${ }^{1}$ and sex workers are therefore at increased risk for cervical cancer. Results of earlier studies showed a higher prevalence of abnormal smears and high risk HPV in sex workers. ${ }^{2}$ In most European countries screening starts at the age of 25 , with the exception of the Netherlands and Finland, where the starting age is 30, and United Kingdom, with screening from 20 years of age. ${ }^{3}$

In Belgium a programme for organised screening has been set up for the Flemish region promoting one cervical smear every 3 years for women aged between 25 and 64 years. ${ }^{4}$ The aim of this study was to assess the prevalence of abnormal smears and HPV in sex workers in Belgium, and to decide whether the recommended screening policy for the early detection of cervical cancer and its precursors is adequate for this specific group of women.

\section{METHODS}

In the outreach programme PASOP, targeted at all sex workers in the region of Ghent (population 1350 000), the health team offers screening for STI, a cervical smear and vaccination against hepatitis $\mathrm{B}$. In this analysis the results of the cervical smears of 653 women tested for the first time in the programme from 1992 until 2001 were used. From 1992 until 1999 the cervical smear was conventional $(n=474)$, but from 2000 it switched to liquid based $(n=179)$. Thin layer cervical slides were prepared with the robotic AutoCyte Prep system according to the manufacturer's instructions. An equal number of controls were selected from the routine screening data of 2001 from Ghent University Hospital (serving the population in the same area as where the sex workers were recruited), matched for age, using liquid based smears. Smear results were classified as negative for intraepithelial lesion or malignancy, atypical glandular cells of undetermined significance (AGUS)/atypical squamous cells of undetermined significance (ASCUS), low grade squamous intraepithelial lesion (LSIL) and high grade squamous intraepithelial lesion (HSIL) according to the Bethesda classification. ${ }^{5}$

Separately from the routine cervical smears, we performed HPV typing on 99 consecutive cervical samples in the screening programme of 2001 to estimate the prevalence of different HPV genotypes. We chose 99 controls from the general population, matched for age, who were routinely screened at Ghent University Hospital in the same period. Samples identified as HPV positive, based on a reverse hybridisation assay, were genotyped. HPV amplimers were identified simultaneously as one of 25 high or low oncogenic risk types, or, if no hybridisation to any probe, assigned as "x." ${ }^{\prime \prime}$

We analysed the results of the cervical smears of the samples with HPV typing. The differences in prevalence of smear results and high risk HPV in sex workers and controls were statistically evaluated with the $\chi^{2}$ test.

\section{RESULTS}

\section{Population characteristics}

The sex worker population (mean age 28.1 years, SD 7.7 years, range 17-58 years) included 258 (39.5\%) women under 25. All professional classes took part, except for street workers (not active in this area). The nationalities were $60.5 \%$ Belgian, $14.4 \%$ French, 5.2\% other western European, 7.6\% sub-Saharan African, $7.4 \%$ northern African, and $4.4 \%$ other. The subgroup for HPV typing had the same characteristics.

\section{Cervical smears}

The sex worker population is significantly different from the control group.

Combining the classes AGUS/ASCUS, LSIL, and HSIL in a group of candidates for follow up (repeat smears, colposcopy), the odds ratio for sex workers belonging to the follow 
Table 1 Smear results for 653 sex workers and 653 age matched controls in Belgium (1992-2001)

\begin{tabular}{lllll}
\hline Smear result & $\begin{array}{l}\text { Sex worker }(\mathbf{n}=653) \\
(95 \% \mathrm{Cl})\end{array}$ & $\begin{array}{l}\text { Control }(\mathbf{n}=653) \\
(95 \% \mathrm{CI})\end{array}$ & OR (95\% CI) & p Value \\
\hline $\begin{array}{l}\text { Negative for } \\
\text { intraepithelial } \\
\text { lesion or }\end{array}$ & $78.9 \%(75.7$ to 82.0$)$ & $95.1 \%(93.4$ to 96.8$)$ & $0.85(0.68$ to 1.06$)$ & 0.13 \\
malignancy & & & & \\
AGUS/ASCUS & $2.6 \%(1.4$ to 3.8) & $1.4 \%(0.5$ to 2.3$)$ & $1.90(0.80$ to 4.67$)$ & 0.11 \\
LSIL & $15.6 \%(12.8$ to 18.4$)$ & $2.9 \%(1.6$ to 4.2$)$ & $6.18(3.65$ to 10.56$)$ & $<0.001$ \\
HSIL & $2.9 \%(1.6$ to 4.2$)$ & $0.6 \%(0.1$ to 1.2$)$ & $4.86(1.55$ to 16.97$)$ & $<0.001$ \\
Follow up group, & $21.1 \%(18.0$ to 24.3$)$ & $4.9 \%(3.2$ to 6.6$)$ & $7.69(4.73$ to 12.59$)$ & $<0.001$ \\
women with & & & & \\
AGUS/ASCUS, & & & & \\
LSIL, or HSIL & & & & \\
\hline
\end{tabular}

up group is 7.7 (95\% confidence interval 4.7 to 12.6) in comparison with women of the same age from the general population. When considering only the women under 25 , $24.4 \%$ were candidate for follow up, in comparison with $5.1 \%$ of the women under 25 in the control group (OR 6.1, 95\% CI 3.2 to 12.0$)$.

\section{HPV typing}

In the sex worker group a total of six results were nonconclusive, 21 (22.6\%) (95\% CI: 14.1 to 31.1 ) were negative, and $72(77.4 \%)$ (95\% CI: 68.9 to 85.9 ) were positive for HPV. The prevalence of HPV types 16, 31 and 52 were highest $(12.9 \%)$.

Of the $21(22.6 \%)$ negative samples, $17 \quad(85 \%)$ were negative for intraepithelial lesion or malignancy, and three ( $15 \%)$ were candidates for follow up (no result for one). Of

Table 2 HPV types for 93 sex workers and 98 age matched controls in Belgium (2001)

\begin{tabular}{|c|c|c|c|}
\hline \multirow[b]{2}{*}{ HPV } & \multirow[b]{2}{*}{ HPV type } & \multirow{2}{*}{$\begin{array}{l}\text { Sex workers } \\
(n=93) \\
\text { No }(\%)\end{array}$} & \multirow{2}{*}{$\begin{array}{l}\text { Controls } \\
\text { ( } n=98) \\
\text { No (\%) }\end{array}$} \\
\hline & & & \\
\hline \multirow[t]{6}{*}{ Negative } & & 21 (22.6) & 71 (72.4) \\
\hline & 6 & $5(5.4)$ & \\
\hline & 11 & $2(2.2)$ & \\
\hline & 34 & $1(1.1)$ & \\
\hline & 40 & 3 (3.2) & \\
\hline & 42 & $1(1.1)$ & \\
\hline \multirow[t]{13}{*}{ Low risk } & 43 & $1(1.1)$ & $1(1.0)$ \\
\hline & 44 & $6(6.5)$ & \\
\hline & 53 & $6(6.5)$ & \\
\hline & 54 & $3(3.2)$ & $1(1.0)$ \\
\hline & 70 & $6(6.5)$ & $2(2.0)$ \\
\hline & 74 & $6(6.5)$ & \\
\hline & $\mathrm{X}$ : benign types & $8(8.6)$ & $8(8.2)$ \\
\hline & 16 & $12(12.9)$ & $4(4.1)$ \\
\hline & 18 & $3(3.2)$ & $2(2.0)$ \\
\hline & 31 & $12(12.9)$ & $1(1.0)$ \\
\hline & 33 & $6(6.5)$ & $1(1.0)$ \\
\hline & 35 & 3 (3.2) & $1(1.0)$ \\
\hline & 39 & $7(7.5)$ & $2(2.0)$ \\
\hline \multirow{8}{*}{ High risk } & 45 & $9(9.7)$ & $2(2.0)$ \\
\hline & 51 & $1(1.1)$ & $1(1.0)$ \\
\hline & 52 & 12 (12.9) & $2(2.0)$ \\
\hline & 56 & $6(6.5)$ & $1(1.0)$ \\
\hline & 58 & $2(2.2)$ & \\
\hline & 59 & $4(4.3)$ & \\
\hline & & 3 (3.2) & $2(2.0)$ \\
\hline & 68 or $73^{*}$ & $10(10.8)$ & \\
\hline
\end{tabular}

*According to manufacturer genotype 68 and 73 may show cross reactivity. the $20(21.5 \%)$ low risk HPV samples, 15 (79.0\%) were negative and four $(21 \%)$ abnormal (no result for one). Of the $52(55.9 \%)$ high risk HPV samples, $34(65.4 \%)$ were negative and $18(34.6 \%)$ abnormal $(\mathrm{p}=0.078)$.

In the control group one result was non-conclusive, 71 (72.4\%) (95\% CI: 63.6 to 81.3 ) were negative, and 27 (27.6\%) (95\% CI: 18.7 to 36.3 ) were positive for HPV (13 (13.3\%) low risk, and 14 (14.3\%) high risk). All samples were negative for intraepithelial lesion or malignancy.

The odds ratio to be HPV positive was 9.0 (95\% CI: 4.5 to 18.5), and the odds ratio to have one or more high risk HPV was 7.6 (95\% CI: 3.6 to 16.3 ) for sex workers in comparison with the controls.

The multiple presence of HPV genotypes was found more often in sex workers $(50 \%)$ than in the controls $(22.2 \%)$ $(\mathrm{p}<0.05)$.

\section{DISCUSSION}

The prevalence of LSIL (15.6\%) and HSIL (2.9\%) at routine screening of sex workers in our study is very high in comparison with a control group of women of the same age and same region, but not in sex work (LSIL 2.9\%, HSIL 0.6\%). Cervical smears of all controls were evaluated using liquid based cytology. Since the sensitivity of Pap smears with this technique is higher, ${ }^{7}$ the real contrast between sex workers and controls may be even larger. The prevalence was similar to a small group of sex workers (97) tested in Ireland ${ }^{8}$ (abnormal smear in 19\%), but much higher than found in Singapore $^{9}$ (5.6\%) and in Austria (6.3\%), ${ }^{2}$ The prevalence of HPV in sex workers is very high too $(77.4 \%)$ compared with the control group $(27.6 \%)$. In comparison to other studies among sex workers (48.9\% in Mexico City, ${ }^{10} 43 \%$ in Senegal, ${ }^{11}$ $14.4 \%$ in Singapore ${ }^{9}$ ), HPV prevalence is highest in our group. The high prevalence of HPV may be explained partly by the higher sensitivity of the applied tests. Since we used the same tests for the controls, however, the results for the sex workers remain significantly higher than expected. Although numbers are low, the results of the cervical smears for the HPV typed samples are consistent, with more pathology for the high risk types.

The results for multiple infections were similar to those found in Mexico (58.3\%). ${ }^{10}$

Almost one quarter, or 63 women of our group of sex workers younger than 25 (258 women), was referred for repeat smears or colposcopy. If the recommendations for Belgium and most European countries had been applied here, these sex workers with abnormal smears would have been detected only with some delay. We therefore propose to offer sex workers a test when they start working in sex work, regardless of their age. The high prevalence of oncogenic HPV 


\section{Key messages}

- Sex workers in Belgium are at increased risk for abnormal cervical smears and HPV in comparison with the general population.

- Cervical cancer screening should start at the onset of sex work, irrespective of age.

in sex workers supports a higher screening frequency for this group-for example, once a year.

We recommend the use of targeted services to implement cervical screening in the sex worker community. In the case of hepatitis B vaccination, targeted services have proved to reach sex workers better than regular health services. ${ }^{12}$

\section{ACKNOWLEDGEMENTS}

The outreach project was supported by the Ministry of Health of Flanders, the Province of East Flanders, and the City of Ghent. PCR typing was supported by the Ghent University. We particularly thank all women for their trust in the project.

\section{CONTRIBUTORS}

RM is the principal investigator, LVR and CC performed and interpreted the tests, and were involved in the redaction of the article.

\section{Authors' affiliations}

R Mak, Department of Public Health, Ghent University, De Pintelaan 185,9000 Ghent, Belgium and PASOP VZW, Sex Workers Health Centre, Brabantdam 100B, 9000 Ghent, Belgium

L Van Renterghem, Laboratory of Bacteriology and Virology, Ghent University Hospital, De Pintelaan 185, 9000 Ghent, Belgium
C Cuvelier, Department of Pathology, Ghent University, De Pintelaan 185,9000 Ghent, Belgium

Correspondence to: Rudolf Mak, MD, Department of Public Health, Ghent University, De Pintelaan 185, 9000 Ghent, Belgium; rudolf.mak@rug.ac.be

Accepted for publication 7 May 2003

\section{REFERENCES}

1 Walboomers JM, Jacobs MV, Manos MM, et al. Human papillomavirus is a necessary cause of invasive cervical cancer worldwide. J Pathol 1999;189:12-19.

2 Gitsch G, Kainz Ch, Reinthaller A, et al. Cervical neoplasia and human papilloma virus infection in prostitutes. Genitourin Med 1991;67:478-80.

3 Linos A, Riza E. Comparisons of cervical cancer screening programmes in the European Union. Eur J Cancer 2000;36:2260-65.

4 Arbyn M, Van Oyen H. Cervical cancer screening in Belgium. Eur J Cancer 2000;36:2191-97.

5 Report of the 1991 Bethesda Workshop. The 1991 Bethesda system for reporting cervical/vaginal cytological diagnoses. JAMA 1992;267:1892.

6 Kleter B, van Doorn U, Schrauwen L, et al. Development and clinical evaluation of a highly sensititve PCR-reverse hybridization line probe assay for detection and identification of anogenital human papillomavirus. J Clin Microbiol 1999:37:2508-17.

7 McDonnell R, McDonneell P, $\mathrm{O}^{\prime} \mathrm{Neill} M$, et al. Health risk profile of prostitutes in Dublin. Int J STD AIDS 1998;9:485-88.

8 Hutchinson ML, Zahniser DJ, Sherman ME, et al. Utility of liquid-based cytology for cervical cancer screening: results of a population-based study conducted in a high cervical cancer incidence region of Costa Rica. Cancer Cytopathol 1999;87:48-55.

9 Chan R, Khoo L, Ho TH, et al. A comparative study of cervical cytology, colposcopy and PCR for HPV in female sex workers in Singapore. Int J STD AIDS 2001;12:159-63.

10 Juarez-Figueroa LA, Wheeler CM, Uribe-Salas FJ, et al. Human papillomavirus. A highly prevalent sexually transmitted disease agent among female sex workers from Mexico City. Sex Transm Dis 2001;28:125-30.

11 Langley C, Benga-De E, Critchlow C, et al. HIV-1, HIV-2, human papillomavirus infection and cervical neoplasia in high-risk African women. AIDS 1996; 10:413-17.

12 Mak R, Traen A, Claeyssens M, et al. Hepatitis B vaccination for sex workers: do outreach programmes perform better? Sex Transm Infect 2003;79:157-9.

\section{$\mathrm{ECHO}$}

\section{Multiple high risk HPV infections and cervical screening}

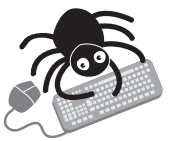

Please visit the Sexually Transmitted Infections website [www. stijournal.com] for a link to the full text of this article.
T wenty per cent of cytology samples from routine cervical screening clinics in Edinburgh, UK, were found to be positive for human papillomavirus (HPV). Multiple high risk HPV (HR-HPV) infections were most prevalent in young women, who usually have higher levels of sexual activity, and in around $40 \%$ of samples with all grades of dyskaryosis.

The addition of HR-HPV testing to cervical screening programmes may enhance the programmes' accuracy by identifying those individuals at greater risk of disease progression. This study showed that the detection of multiple HR-HPV was not a significantly better predictor of high grade cervical neoplasia being no more frequent in high grade than low grade dyskaryosis. The finding probably reflects the common sexual transmission of multiple HR-HPV types together.

Participating clinics in Edinburgh used a liquid based cytology method which has the advantage that the residual suspension not used for cytology can be used for microbiological testing. The extent of multiple infection was assessed in residual material from 3444 liquid based cytology samples using real time GP5+/GP6+ polymerase chain reaction for screening, and linear array assay for genotyping.

Future prospective cohort studies that link sequential loss or gain of HPV types with cytological analysis will be able to assess the impact of multiple HR-HPV infections on neoplastic progression.

A Journal of Clinical Pathology 2004;57:68-72. 